\title{
Analysis of the Hysteresis Behavior of Perovskite Solar Cells with Interfacial Fullerene Self-Assembled Monolayers
}

\author{
Marta Valles-Pelarda, ${ }^{1}$ Bruno Clasen Hames, ${ }^{1}$ Inés García-Benito, ${ }^{2,3}$ Osbel Almora, ${ }^{1}$ \\ Agustin Molina-Ontoria, ${ }^{2}$ Rafael S. Sánchez, ${ }^{1}$ Germa Garcia-Belmonte, ${ }^{1}$ Nazario \\ Martín $^{2,3, *}$ and Ivan Mora-Sero ${ }^{1, *}$
}

\author{
${ }^{1}$ Institute of Advanced Materials (INAM), Universitat Jaume I, Av. Sos Baynat, s/n, \\ 12006 Castellón, Spain \\ 2 IMDEA-Nanociencia, C/Faraday 9, Campus de la Universidad Autónoma de Madrid, \\ E-28049 Madrid, Spain \\ ${ }^{3}$ Departamento de Química Orgánica. Facultad de Ciencias Químicas, Universidad \\ Complutense de Madrid, E-28040 Madrid, Spain \\ Corresponding authors: nazmar@ucm.es, sero@uji.es
}

\begin{abstract}
The use of self-assembled monolayers (SAM) of fullerene derivatives reduces the hysteresis of perovskite solar cells (PSCs). We have investigate three different fullerene derivatives observing a decrease on hysteresis for all the cases. Several processes can contribute to the hysteresis behavior on PSCs. We have determined that the reduced hysteresis observed for devices with SAM is produced by a decrease of the capacitive hysteresis. In addition, with an appropriated functionalization SAM can increase photocurrent even when no electron selective contact (ESC) is present and SAM is deposited just on top of the transparent conductive oxide. Appropriated functionalization of the fullerene derivative, as introducing -CN groups, can enhance cell performance and reduce hysteresis. This work paves the way for a future enhancement of PSCs by a tailored design of the fullerene molecules that could actuate as an ESC by themselves.
\end{abstract}


TOC Figure

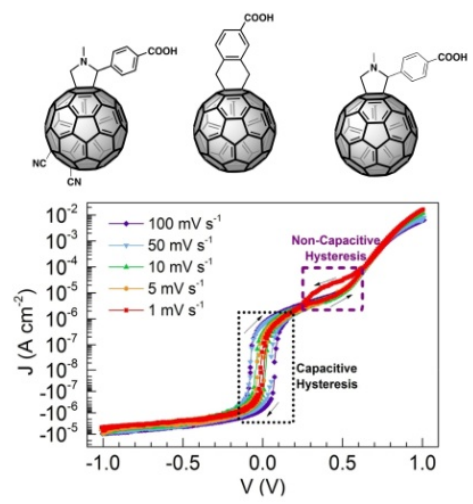


After the first reports on all-solid perovskite solar cells (PSCs), ${ }^{1,2}$ the interest of the scientific community on this kind of devices has boosted. Successive improvements of the cell configuration, deposition methods, perovskite composition (combination of organic and inorganic cations with halide anions) as well as hole and electron transporting materials, ${ }^{3-7}$ have produce record certified devices with efficiencies as high as $22.1 \%{ }^{8}$ Nevertheless, despite the spectacular enhancement in very few years, many aspects related to the behavior of halide perovskites and to the working principles of PSCs remain unclear and are still under debate. One of these debates is the role of the charge selective contacts, not only in terms of the overall efficiency but also in terms of the anomalous phenomena widely observed in PSCs; for instance, the hysteresis present in the Current-Potential (J-V) curves. Many different contacts have been studied for PSCs, which show their important role on the solar cell efficiency, stability and hysteresis. ${ }^{9,10}$ It is commonly established that the use of fullerene derivatives in PSCs with inverted configuration produce devices without J-V hysteresis; however, recent papers demonstrate that this statement cannot be generalized, for example significant hysteresis has been detected at low temperature even with the presence of fullerene derivatives. ${ }^{11,12}$ Hysteresis in inverted configuration devices appears in different conditions and shows different and using $\mathrm{TiO}_{2}$ mesoporous scaffold. ${ }^{11,13,14}$ In this communication, we have studied the effect of a Self-Assembled Monolayer (SAM) of different fullerene derivatives specifically functionalized to anchor to an oxide surface. The effect of the anchoring moiety, the insertion of additional functional groups in the fullerene unit for a more intimate contact with the perovskite and the role of compact selective contacts have been analyzed by focusing on the solar cell performance and hysteresis. 
Very recently, capacitive and non-capacitive components, whose origins are believed to arise from different physical and/or chemical mechanisms, have been identified in the hysteresis of PSCs. ${ }^{14}$ Capacitive hysteresis is directly proportional to the Current-Potential (J-V) curve scan rate, s. A capacitor with capacitance $C$ presents a measured current $s \cdot C$ in the $\mathrm{J}-\mathrm{V}$ curve observed during the voltage sweep. The change of the scan rate direction produces consequently a change in the sign of the current provoking the hysteresis. Moreover non-capacitive hysteresis is not proportional to scan rate and has a different physical origin as we discuss below. We have observed that perovskite solar cells (PSCs) prepared with a SAM of fullerene derivatives present a mixed hysteresis behavior; firstly, they exhibit the typical capacitive component of a standard PSCs prepared with $\mathrm{TiO}_{2}$ as an electron selective contact (ESC), although the hysteresis is significantly less pronounced when the fullerene SAM is introduced; secondly, a non-capacitive hysteresis previously observed also in inverted PSCs with fullerene was also detected in PSCs with a SAM.

The photovoltaic performance does not only depend on the light absorbing material but also on the efficient charge extraction at the selective contacts. ${ }^{15} \mathrm{TiO}_{2}$ is probably the most extensively used ESC in PSCs, although organic ESC are receiving an increasing attention. ${ }^{16}$ In accordance with this, it has been shown that the use of fullerene derivative layers, ${ }^{17,18}$ the deposition of a thin fullerene layer ${ }^{19,20}$ or even the use of a SAM on top of a $\mathrm{TiO}_{2}{ }^{21}$ reduces significantly the photoluminescence lifetime, thus indicating a better electron extraction when the fullerene derivatives substitute the $\mathrm{TiO}_{2}$ or even when the fullerene is on the top of the $\mathrm{TiO}_{2}$ layer. Therefore, modifying the ESC-perovskite interface can significantly enhancethe electron injection and electron transfer, as well as reducing the charge recombination. ${ }^{19,21}$ As it has previously been commented, the approach that consists in functionalizing the ESC with fullerene 
derivatives has been investigated in this work. Fullerenes have high electron affinity and are good electron acceptors. Their $\pi$-conjugated structure enables the charge delocalization, thus enhancing the electron extraction. As it has already been mentioned, fullerenes have reduced the hysteresis effect observed in PSCs from the standard J-V measurements. In this sense, it is especially interesting to analyze how a single fullerene monolayer can affect the ESC-perovskite interface properties. It is worth pointing out that such interesting approach constitutes a valuable tool for a better understanding of the hysteresis origins and its subsequent minimization, while it is based on easy and material saving procedures compared to the approach based on the exploitation of thin layer of a fullerene derivative. Additionally, we have also analyzed how the SAM of the fullerene derivatives behave when no compact $\mathrm{TiO}_{2}$ layer is employed; in particular, we have observed that the deleterious effect of removing the compact $\mathrm{TiO}_{2}$ can be relatively mitigated by the presence of the SAM. The presence of compact $\mathrm{TiO}_{2}$ and/or fullerene derivative SAM has a huge impact on the J-V hysteresis and an appropriate choice of the fullerene derivative can avoid the photocurrent reduction observed when no compact $\mathrm{TiO}_{2}$ is used.

In this work, we have analyzed three different fullerene derivatives (1-3), see Figure 1a, whose syntheses is described in detail in the experimental section. The fullerene derivatives have been functionalized with a carboxyl group (-COOH), which is a widely employed functional group for anchoring to the $\mathrm{TiO}_{2}$ surface. We have selected as a reference the fullerene derivative $\mathbf{3}$, already reported in the literature, ${ }^{21}$ where a benzoic acid is linked to the fullerene through a N-methylpyrrolidine, and we have prepared a couple of variations by changing, on the one hand, the connection between the fullerene and the benzoic acid (using a cyclohexane instead of a pyrrolidine). On the other hand, we have introduced two cyanide groups in the fullerene 
moiety, which significantly modify the reduction potential values of the molecule and provide further anchoring group through the lone pair nitrogen atoms, see Figure 1a. These modifications have allowed us to study not only the influence of the bridging functional group that connects the fullerene with the oxide substrate, but also the influence of the functional groups that contact directly with the perovskite layer, i.e. the cyanide groups from 1, see Figure 1a.The optical absorption of the fullerene derivatives has been analyzed, see Figure S1; very similar features have been observed and a band gap of $\sim 2 \mathrm{eV}$ has been estimated for all three fullerene derivatives, see Table S1. In addition, the HOMO and LUMO energies and the redox potentials, see Table S2, have been measured using cyclic voltammetry, see Figure S2.

(a)

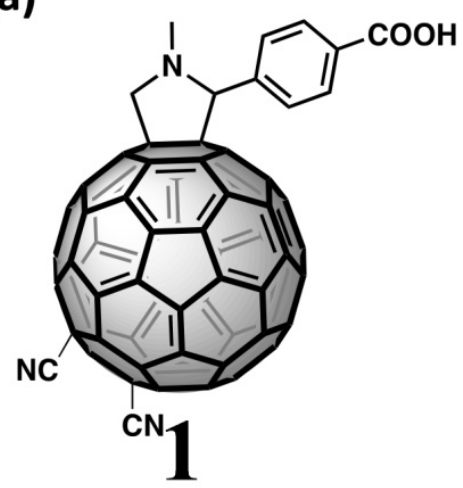

(b)

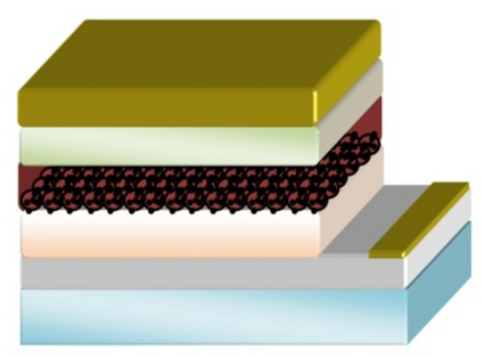

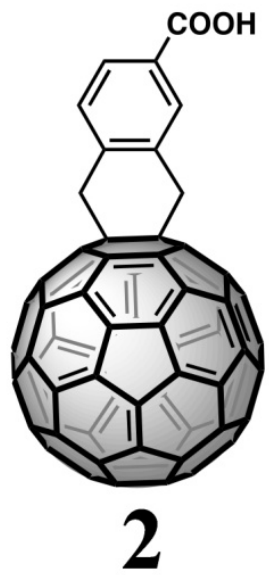

Gold SPPRO-OMeTAD $\mathrm{CH}_{3} \mathrm{NH}_{3} \mathrm{Pbl}_{3-x} \mathrm{Cl}_{x}$

SAM

NS- $-\mathrm{TIO}_{2}$

$\mathrm{CL}-\mathrm{TIO}_{2}$

FTO

Glass

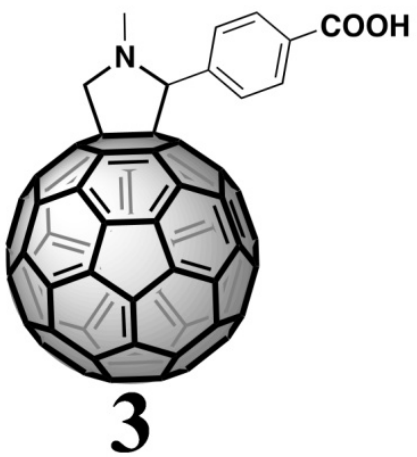

(c)

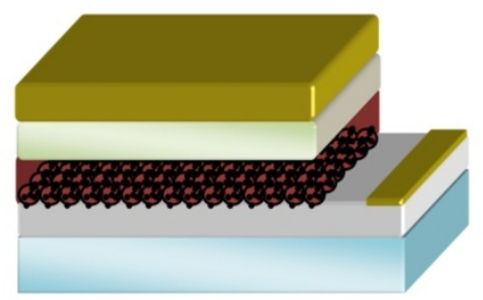

Figure 1: a) Functionalized fullerenes studied in this work. Configuration of the perovskite solar cell analyzed in this work, devices b) with (CL) and c) without (NCL) $\mathrm{TiO}_{2}$ compact layer on top of FTO have been analyzed. Nanostructured $\mathrm{TiO}_{2}$ layer (NS$\mathrm{TiO}_{2}$ ) was used for all the samples. 
In order to analyze the effect of the fullerene derivatives we have selected the probably most extended configuration for PSCs, see Figure 1b. In this configuration a Compact Layer (CL) of $\mathrm{TiO}_{2}$ is deposited on top of a glass/FTO substrate. A mesoporous layer of $\mathrm{TiO}_{2}$ has been subsequently deposited. The deposited perovskite $\mathrm{CH}_{3} \mathrm{NH}_{3} \mathrm{PbI}_{3}$, hereafter $\mathrm{MAPbI}_{3}$, not only infiltrates into the mesoporous layer of $\mathrm{TiO}_{2}$ but also produces a perovskite capping layer on top, see Figure S3. Finally, spiroOMeTAD as a Hole Selective Contact (HSC) and a gold layer are deposited in order to extract efficiently the photo-generated holes. A similar configuration but with $\mathrm{No}^{\mathrm{TiO}} 2$ Compact Layer (NCL) has been also studied, see Figure 1c. Reference samples have been produced by the direct deposition of perovskite on the bare substrates, while devices with interfacial fullerene derivative SAM have been produced by dipping the $\mathrm{TiO}_{2}$ substrates in a chlorobenzene solution containing the desired fullerene derivative during 24 hours, see the experimental section for more details. After the SAM deposition, the surface nature of the substrate is clearly modified, which is manifested through a greater hydrophobicity of the surface.

The effect of using $\mathbf{2}$ and $\mathbf{3}$ derivatives on the cell performance can be observed in the J-V curves under 1 sun illumination, plotted in Figure 2a and 2b, respectively. The reference sample, using the configuration reported in Figure 1b with compact layer and without SAM, is denoted as CL sample. Samples with 2 and 3 SAM are called CL/2 (Figure 2a) and CL/3 (Figure 2b), respectively. The averaged values of the solar cell parameters are summarized in Table 1. It can be clearly observed that the most conspicuous effect of the presence of the SAM is a clear reduction of the hysteresis. The properties of the $\mathrm{TiO}_{2}$-perovskite interfaces have been modified after SAM deposition, as can be clearly observed from the strong quenching of the photoluminescence (PL), 
see Figure 2c, conventionally attributed to an improved charge extraction. ${ }^{21}$ However, in contrast to previous reports, ${ }^{21}$ we have not detected any increase of the photocurrent, which is not altered or is even slightly reduced when the fullerene SAM is introduced, see Figure $2 \mathrm{a}$ and $2 \mathrm{~b}$ respectively. In addition, the slight decrease of $\mathrm{V}_{\text {oc }}$ detected for samples with SAM and the larger dark current detected when SAM is used, in some cases (see Figure S4), makes that it is not possible to discard an increase of the recombination when SAM is used.
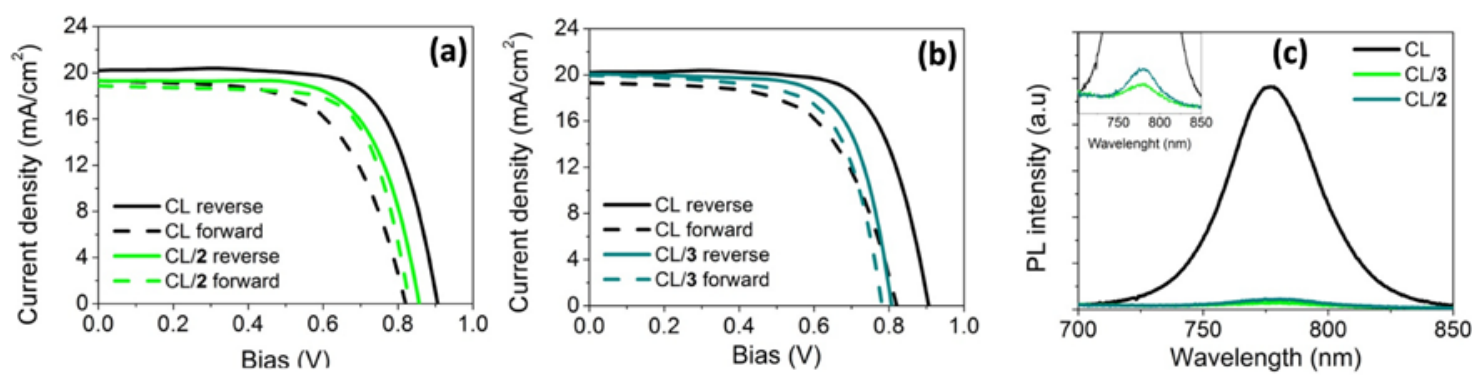

Figure 2: Current-voltage curves for forward and reverse scans for perovskite solar cell with a SAM of a) 2 and b) 3. c) Photoluminescence intensity of reference sample and samples with SAM of 2 and 3.

Table 1. Characteristic parameters of the solar cells analyzed in this work: short circuit current, $\mathrm{J}_{\mathrm{sc}}$, open circuit voltage, $\mathrm{V}_{\mathrm{oc}}$, fill factor, $\mathrm{FF}$, and photoconversion efficiency, $\eta$. Averaged values and the corresponding standard errors have been calculated using the results obtained by at least 10 cells prepared at each condition, see Table S3 and S4. Two different set of samples have been prepared with their corresponding reference samples, in order to compare samples produced with the same experimental conditions. Samples were prepare under air atmosphere and temperature and humidity conditions vary from batch to batch. Consequently we compare samples containing SAM with reference samples produced in the same batch. The hysteresis index (HI) was calculated using Eq. 1 for the J-V curves shown in Figure 2 and 3. 


\begin{tabular}{|c|c|c|c|c|c|c|}
\hline Device & Scan direction & $\mathrm{J}_{\mathrm{sc}}\left(\mathrm{mA} / \mathrm{cm}^{2}\right)$ & $\mathrm{V}_{\mathrm{oc}}(\mathrm{mV})$ & FF (\%) & $\eta(\%)$ & HI \\
\hline \multirow{2}{*}{ CL } & reverse & $19.8 \pm 0.6$ & $909 \pm 15$ & $75.4 \pm 2.1$ & $13.5 \pm 0.6$ & \multirow{2}{*}{$0.067 \pm 0.004$} \\
\hline & forward & $19.3 \pm 0.6$ & $827 \pm 18$ & $63.4 \pm 2.2$ & $10.1 \pm 0.7$ & \\
\hline \multirow{2}{*}{ CL/3 } & reverse & $19.2 \pm 0.5$ & $822 \pm 13$ & $71.4 \pm 1.9$ & $11.2 \pm 0.3$ & \multirow{2}{*}{$0.019 \pm 0.005$} \\
\hline & forward & $18.7 \pm 0.8$ & $797 \pm 15$ & $70.3 \pm 2.0$ & $10.47 \pm 0.22$ & \\
\hline \multirow{2}{*}{ CL/2 } & reverse & $19.2 \pm 0.6$ & $839 \pm 12$ & $67 \pm 4$ & $10.9 \pm 0.8$ & \multirow{2}{*}{$0.036 \pm 0.003$} \\
\hline & forward & $18.7 \pm 0.5$ & $818 \pm 8$ & $66 \pm 5$ & $10.0 \pm 0.8$ & \\
\hline Device & Scan direction & $\mathrm{J}_{\mathrm{sc}}\left(\mathrm{mA} / \mathrm{cm}^{2}\right)$ & $\mathrm{V}_{\mathrm{oc}}(\mathrm{mV})$ & FF (\%) & Eff (\%) & \\
\hline \multirow{2}{*}{ CL } & reverse & $17.1 \pm 1.3$ & $792 \pm 18$ & $68 \pm 5$ & $9.1 \pm 0.9$ & \multirow{2}{*}{$0.20 \pm 0.07$} \\
\hline & forward & $14.5 \pm 2.2$ & $728 \pm 54$ & $49 \pm 9$ & $5.4 \pm 1.7$ & \\
\hline \multirow{2}{*}{ NCL } & reverse & $11.9 \pm .1 .7$ & $817 \pm 16$ & $64 \pm 5$ & $6.2 \pm 1.0$ & \multirow{2}{*}{$0.32 \pm 0.03$} \\
\hline & forward & $11.2 \pm 1.8$ & $768 \pm 35$ & $41 \pm 3$ & $3.6 \pm 0.7$ & \\
\hline \multirow{2}{*}{ CL/1 } & reverse & $18.1 \pm 1.3$ & $780 \pm 11$ & $63 \pm 13$ & $8.9 \pm 1.9$ & \multirow{2}{*}{$0.092 \pm 0.016$} \\
\hline & forward & $16.6 \pm 0.9$ & $779 \pm 6$ & $71.4 \pm 2.1$ & $9.2 \pm 0.5$ & \\
\hline \multirow{2}{*}{ NCL/1 } & reverse & $17.4 \pm 1.9$ & $753 \pm 17$ & $64 \pm 6$ & $8.4 \pm 1.6$ & \multirow{2}{*}{$0.198 \pm 0.014$} \\
\hline & forward & $14 \pm 3$ & $708 \pm 61$ & $43 \pm 6$ & $4.4 \pm 1.3$ & \\
\hline
\end{tabular}

The observed hysteresis has been quantified by calculating the HI as follows: ${ }^{22}$

$H I=\frac{J_{\text {rev }}\left(\mathrm{V}_{O C} / 2\right)-J_{f o r}\left(V_{O C} / 2\right)}{J_{\text {rev }}\left(V_{O C} / 2\right)}$

Where $\mathrm{J}_{\text {rev }}\left(\mathrm{V}_{\text {oc }} / 2\right)$ and $\mathrm{J}_{\text {for }}\left(\mathrm{V}_{\mathrm{oc}} / 2\right)$ are the currents when half of the $\mathrm{V}_{\mathrm{oc}}$ voltage is applied for reverse (from $V_{\text {ос }}$ to zero) and for forward (from zero to $V_{o c}$ ) voltage scans, respectively. A device without hysteresis presents a HI of zero while higher values of HI indicate a more pronounced hysteresis. When 2 and 3 SAM are used the HI is significantly reduced, 2 and 4-fold, respectively.

In order to get further insights about the particularities of the observed hysteresis for samples with fullerene derivatives, J-V curves under dark with different scan rates have been measured, see Figure 3. The hysteresis observed in PSCs has not a single and general origin for all the cases. Very recently, it has been shown that the $\mathrm{J}-\mathrm{V}$ hysteresis under dark of standard devices using $\mathrm{TiO}_{2} /$ spiro-OMeTAD as ESC/HSC has a capacitive origin that is directly proportional to the scan rate. ${ }^{14,23}$ This hysteresis is observed at low applied voltages and we have also observed it when the fullerene 
derivative SAMs are deposited, see dotted frame in Figure 3, and its dependence with the scan rate in the inset of Figure 3. Additionally, we have also observed another hysteresis feature at applied voltages of around $0.5 \mathrm{~V}$ at very low scan rates, and consequently this is believed to arise from a non-capacitive process, see dashed frame in Figure 3. Inverted non-capacitive hysteresis under dark has been observed for inverted PSCs using PEDOT/PCBM as HSC/ESC; however, the non-capacitive hysteresis is still observed for standard PSCs with $\mathrm{TiO}_{2}$ contact, but it is in general less evident. It has been suggested that the origin of non-capacitive hysteresis could be related to the reactivity of the perovskite at the interface with the contact. ${ }^{14,24}$ This non-capacitive hysteresis has been also observed in devices in which neither $\mathrm{TiO}_{2}$ nor organic electron conductors played a role as symmetrical Au/perovskite/Au samples and explained in that case by the formation of blocking contacts at the perovskite/Au interface and the modification of these contact properties following strong polarization. ${ }^{25}$ A more systematic study will be needed to unveil the single o multiple origin of non-capacitive hysteresis in each case. While capacitive hysteresis just difficult the determination of solar cell parameters and will not have any effect at cell working DC conditons, the determination of the physical origin/s of non-radiative hysteresis could have important implications in PSCs. It will be especially important if the physical origin is related with surface reactivity as it could limit the long term solar cell stability.

Interestingly, the observed non-capacitive hysteresis behavior has an inverted nature, first observed by Almora et al., ${ }^{14}$ compared to the capacitive hysteresis, see black arrows in Figure 3. Moreover, very recently, inverted hysteresis under illumination has been also reported for mixed-halide mixed-organic cation perovskites and in $\mathrm{MAPbI}_{3}$ devices with a mesoporous $\mathrm{TiO}_{2}$ scaffold covered with a thin insulating $\mathrm{Al}_{2} \mathrm{O}_{3}$ shell, and its origin is attributed to an energetic extraction barrier at the $\mathrm{TiO}_{2}$ 
interface with dipole layer formation. ${ }^{26}$ In both cases a clear interfacial nature of the phenomena is pointed out and further research will be needed to clarify whether the origin of the inverted hysteresis arises from the same or, on the contrary, from different effects depending on the nature of the contact.

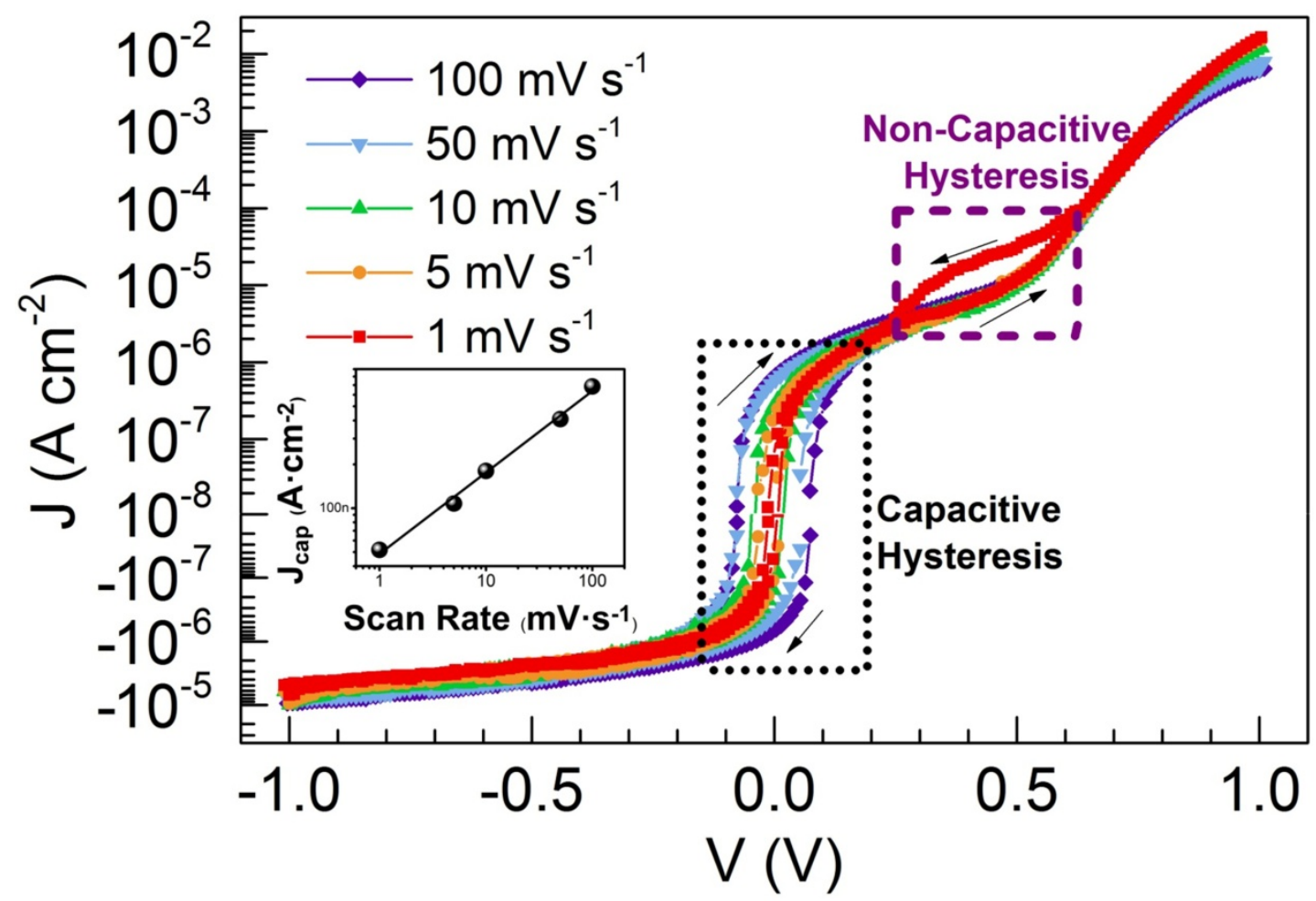

Figure 3: Dark J-V curves in logarithm scaled current representation at different scan rates for a perovskite solar cell with a SAM of 3. Capacitive hysteresis dependent of the scan rate is highlighted with a dotted frame, while Non capacitive hysteresis nonobserved at high scan rates is highlighted with a dashed frame. Black arrows indicate the scan direction. Inset: capacitive current, $\mathrm{J}_{\text {cap }}$, vs. scan rate, symbols represent the experimental points while solid line is the linear fit. $J_{\text {cap }}$ is the contribution of the capacitive current. ${ }^{14}$

Consequently we can conclude that the decrease of the hysteresis observed when fullerene derivative SAMs are employed is due to a decrease of the capacitive hysteresis 
observed for PSCs with $\mathrm{TiO}_{2}$ as ESC. ${ }^{14}$ Moreover, for samples with fullerene SAM is observed the non-capacitive hysteresis of the $\mathrm{J}-\mathrm{V}$ curve under dark also observed for low scan rates in inverted PSCs with organic selective contacts and in devices with $\mathrm{TiO}_{2}$ as ESC. ${ }^{14}$ To this extent, PSCs with fullerene derivative SAM is located half way between both types of PSCs, as present lower capacitive hysteresis than samples with $\mathrm{TiO}_{2}$ contact and higher capacitive hysteresis than inverted cells with organic contacts where capacitive hysteresis is practically negligible.

Moreover, the variation of the bridging group between the fullerene and the benzoic acid, fullerenes 2 and 3, does not introduce any significant change on the performance of the PSCs, see Figure 2. Compounds 2 and $\mathbf{3}$ present the same bandgap, see Table S1, and LUMO position, see Table S2, and induce a PL quenching of the perovskite quite similar, thus indicating that comparable charge injecting properties are induced; therefore the effect of the connecting cyclohexane and pyrrolidine groups induces a negligible effect on the final cell performance. However, the properties induced by the fullerene derivative $\mathbf{1}$ are significantly changed from the reference fullerene $\mathbf{3}$ when the connecting part containing the carboxylic group is kept unchanged but a couple of cyanide groups are introduced into the fullerene framework, see Figure 1a. Cyanide are electron withdrawing groups that cause a higher polarization of the molecule. Cyanide groups does not significantly change the bandgap compared to the $\mathbf{3}$ derivative, see Table S1, but they have a strong effect on the LUMO energy position, which decreases in 20-14 meV compared to the 2/3 counterparts, respectively, see Table 2.

A new set of samples has been prepared using a 1 SAM, see Table 1 and Figure 4a. Again, the use of a SAM reduces the J-V curve hysteresis, but with derivative 1 the efficiency of the PSC increases mainly due to the increase of the measured 
photocurrent. Note that for scan rates in the order of the one used in this work (50 $\mathrm{mV} / \mathrm{s}$ ) the stabilized photocurrent lies between forward and reverse curves. ${ }^{27}$ The origin of this beneficial effect could be ascribed to the direct contact between the highly polar cyanide groups and the perovskite layer, thus allowing an enhanced coupling of the fullerene-perovskite interface. However, an effect of SAM that changes the wettability of the substrate affecting the morphology of the perovskite layer ${ }^{28}$ needs a detailed study beyond the scope of this manuscript. In addition, a strong PL quenching of the perovskite emission has been also observed when the 1 SAM is used, see Figure S5. Note that when the compact layer is removed a partial PL quenching it is also observed, see Figure S5. This fact can be attributed to an increase of surface recombination as the increase of dark current points out, see Figure S4. However, the PL quenching when SAM is added is stronger thus indicating a significant enhancement of the charge carrier injection. An improved design of the anchoring groups of the fullerene derivatives, as well as the coupling enhancement of the fullerene-perovskite interface induced by the introduction of compatible functional groups, e.g. cyanide groups, provide a broad range of possibilities for the exploration of the PSCs performance by means a simple approach consisting in the insertion of an interfacial SAM layer. 

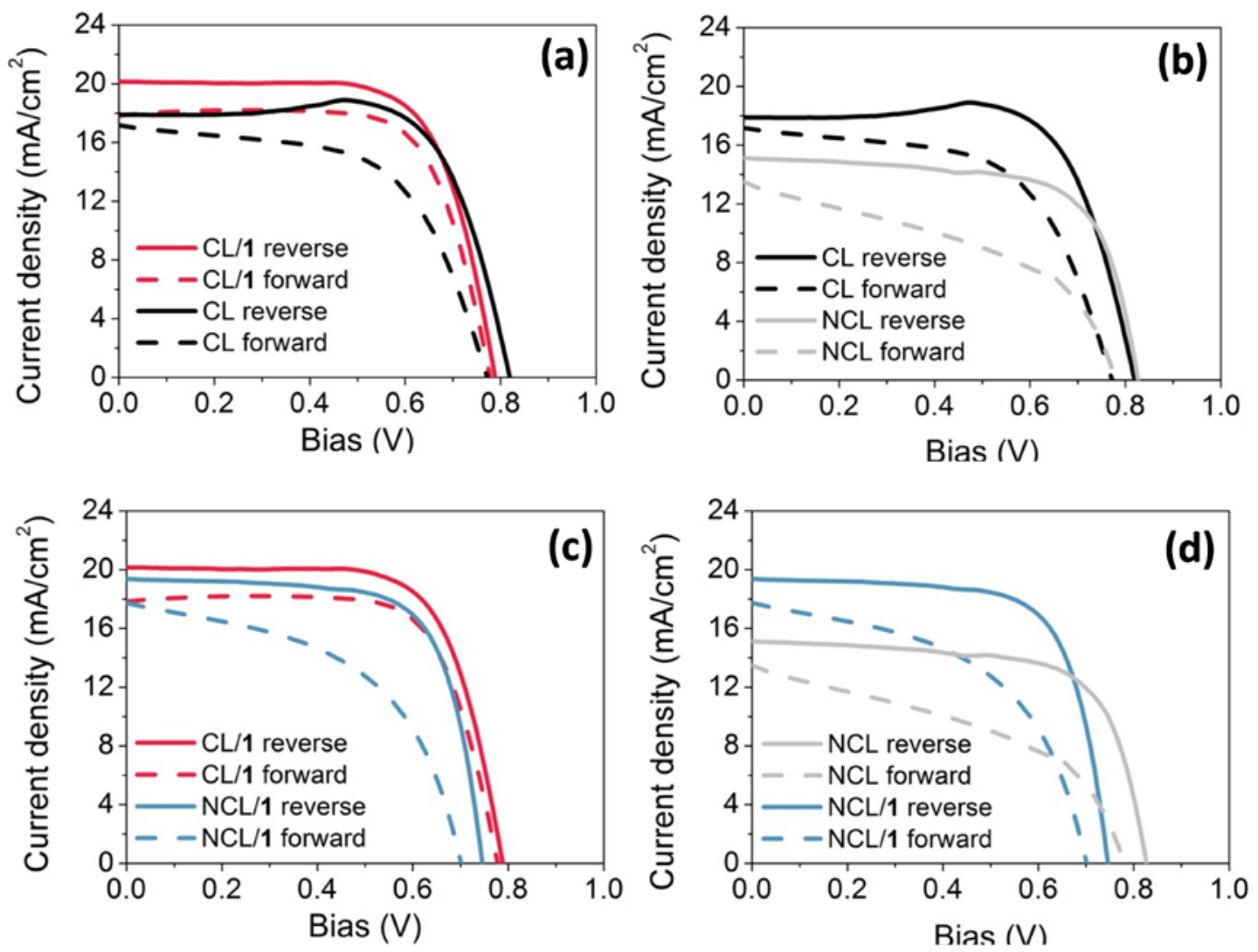

Figure 4: J-V curves for forward and reverse scans under 1 sun illumination comparing samples with and without fullerene 1 SAM. a) Perovskite solar cells with compact layer (CL) of $\mathrm{TiO}_{2}$ with and without a SAM of 1. b) Perovskite solar cells without SAM comparing samples with compact layer (CL) of $\mathrm{TiO}_{2}$ and with no compact layer (NCL) of $\mathrm{TiO}_{2}$. c) Perovskite solar cells with SAM of 1 comparing samples with compact layer (CL) of $\mathrm{TiO}_{2}$ and with no compact layer (NCL) of $\mathrm{TiO}_{2}$. d) Perovskite solar cells with no compact layer (CL) of $\mathrm{TiO}_{2}$ comparing samples with and without a SAM of $\mathbf{1}$.

Finally, we have studied the effect of removing the compact $\mathrm{TiO}_{2} \mathrm{ESC}$, but maintaining the $\mathrm{TiO}_{2}$ scaffold. When the ESC is removed the efficiency of the device is severely affected, see Table 1 and Figure 4b, mainly due to the strong decrease of photocurrent, indicating the important role of the compact layer. A detailed analysis of the scaffold effect is beyond the scope of this work. We have already reported that ESC 
plays a fundamental role in the control of interfacial recombination. ${ }^{29}$ Nevertheless, when 1SAM is employed, there is practically no current decrease, see Figure 4c, due to the interfacial passivation effect induced by the SAM. The use of SAM reduced the hysteresis even when no ESC is present, see Fig. 4d and HI in Table 1. However, in the case of NCL samples the reduction of hysteresis induced by the presence of SAM is significantly lower, see Figure 4d, than in the case of samples with ESC. Several reasons could affect the efficiency of SAM for hysteresis mitigation when no ESC is present: the reactivity of the surface is different, the monolayer quality on FTO may not be as good as on $\mathrm{TiO}_{2}$ or probably the charge transfer between fullerene and FTO or $\mathrm{TiO}_{2}$ could differ significantly. Further analysis is needed to clarify this important question that relates contact nature and hysteresis effect. The use of an appropriated fullerene derivative as $\mathbf{1}$ can significantly mitigate the impact of removing the CL on the $\mathrm{J}_{\mathrm{sc}}$; further mitigation of the hysteresis effect by exploring suitable modifications of the fullerene derivative could allow the complete removal of the inorganic ESC, which could provide high impact benefits from the industrial point of view, as it will permit a simplification of the device configuration and processing, for instance it would avoid a high temperature step in the preparation of PSCs.

In summary, we have analyzed PSCs with different [60]fullerene derivatives SAMs at the ESC-perovskite interface, which induces in all the cases an important reduction of the $\mathrm{J}-\mathrm{V}$ curve hysteresis. We have determined that the hysteresis reduction is due to the decrease of the capacitive hysteresis typically observed for oxide-based anodes in PSCs. SAM samples also present non-capacitive hysteresis in the dark J-V curve, observed at low scan rates, typically observed in inverted PSCs with organic contacts and in lower extend in standard PSCs with compact $\mathrm{TiO}_{2}$ ESC. To this extend PSCs with SAMs of fullerene derivatives are mid way devices between standard and 
inverted PSCs. In addition, we have determined that the design of new fullerene derivatives plays a very important role in the overall solar cell performance, as it has been demonstrated by the inclusion of cyanide groups that enhance the direct coupling with the perovskite layer, thus improving the solar cell parameters, as photocurrent, compared to the reference device with no SAM. However, other aspects as the increase of recombination need to be controlled to optimize the system. The beneficial effect of the SAM can even be extended to the complete removal of the ESC. We have observed that only a slight reduction of the hysteresis is observed when 1 SAM is used with no compact $\mathrm{TiO}_{2}$ layer; the $1 \mathrm{SAM}$ passivates the FTO surface, thus maintaining the device photocurrent. This work explains the origin of the hysteresis reduction when a fullerene derivative SAM is employed and paves the way for a future enhancement of PSCs by means of a tailored design of the fullerene molecules that could actuate as an ESC by themselves, without further need of a $\mathrm{TiO}_{2}$ compact layer.

\section{Methods}

Chemicals: Titanium diisopropoxidebis(acetylacetonate), lead chloride ( $\left.\mathrm{PbCl}_{2}\right)$, dimethylformamide (DMF), anhydrous chlorobenzene, lithium bis(trifluoromethylsulphonyl)imide (Li-TFSI) and 4-tert-butylpiridine (TBP) were purchased from Sigma-Aldrich. $\mathrm{TiO}_{2}$ 18NRT paste was purchased from Dyesol and Methylammonium iodide (MAI) was purchased from TCI. All the materials were used as received. The different fullerene derivatives were synthesized at IMDEA Institute as detailed below.

Synthesis of [60]fullerenes derivatives: [60]Fullerene 3.A mixture of 4Carboxybenzaldehyde (104 mg, $0.70 \mathrm{mmol}), \mathrm{C}_{60}(100 \mathrm{mg}, 0.14 \mathrm{mmol})$ and sarcosine (25 mg, $0.28 \mathrm{mmol}$ ) were dissolved in toluene and the mixture was refluxed for 5 hours. 
The reaction mixture was allowed to reach room temperature and the solvent was removed under vacuum. The crude was purified by flash column chromatography on $\mathrm{SiO}_{2}$, using $\mathrm{CS}_{2} /$ Toluene and then Toluene/ethyl acetate (2:1). The black solid obtained was further purified by repeated $(3 \mathrm{x})$ precipitation and centrifugation in methanol to yield the corresponding hybrids as black solids. ${ }^{1} \mathrm{H}$ NMR (DMSO- $\mathrm{d}_{6}, 300 \mathrm{MHz}, 298 \mathrm{~K}$ ) $\delta 13.0(\mathrm{~s}, 1 \mathrm{H}), 8.04(\mathrm{~d}, \mathrm{~J}=7.6 \mathrm{~Hz}, 2 \mathrm{H}), 7.94(\mathrm{~d}, \mathrm{~J}=7.8 \mathrm{~Hz}, 2 \mathrm{H}), 5.22(\mathrm{~s}, 1 \mathrm{H}), 5.11(\mathrm{~d}, \mathrm{~J}$ $=9.4,1 \mathrm{H}), 4.35(\mathrm{~d}, \mathrm{~J}=9.4 \mathrm{~Hz}, 1 \mathrm{H}), 3.17(\mathrm{~s}, 3 \mathrm{H})$.

[60]Fullerene 2. In a dried $500 \mathrm{~mL}$ round bottom flask, $358 \mathrm{mg}(0.497 \mathrm{mmol})$ of $\mathrm{C}_{60}$ was dissolved in $250 \mathrm{~mL}$ of dry toluene and sonicated for $15 \mathrm{~min}$. 3,4Bis(bromomethyl)benzoic acid (156.2 mg $0.507 \mathrm{mmol}$ ), potassium iodide (585 mg 3.5 mmol), and 18-Crown-6 (250 mg $0.94 \mathrm{mmol}$ ) were sequentially added while stirring under dry nitrogen. The reaction mixture was stirred and heated at reflux for 14 hours. The reaction mixture was allowed to reach room temperature and the solvent was removed under vacuum. The crude was purified by flash column chromatography on $\mathrm{SiO}_{2}$ using $\left(\mathrm{CS}_{2} /\right.$ Toluene and then Toluene/THF (2:1)). The black solid obtained was further purified by repeated (3x) precipitation and centrifugation in methanol to yield the corresponding hybrid as black solid. ${ }^{1} \mathrm{H}$ NMR (DMSO-d $\left.{ }_{6}, 300 \mathrm{MHz}, 298 \mathrm{~K}\right) \delta 13.1$ (s, 1H), 8.35 (d, J = 1.6 Hz, 1H), 8.14 (dd, J = $7.8 \mathrm{~Hz}, \mathrm{~J}=1.6 \mathrm{~Hz}, 1 \mathrm{H}), 7.90$ (d, J = 7.8 Hz, 1H), 5.06 (m , 2H), $4.66(\mathrm{~m}, 2 \mathrm{H})$.

[60]Fullerene 1. A mixture of 4-Carboxybenzaldehyde (14.2 mg, $0.09 \mathrm{mmol}), \mathrm{C}_{60}-\mathrm{CN}_{2}$ (80 mg, $0.10 \mathrm{mmol}$ ) and sarcosine (41.4 $\mathrm{mg}, 0.47 \mathrm{mmol}$ ) were dissolved in chlorobenzene (20 mL) and the mixture was refluxed for 20 hours. The reaction mixture was allowed to reach room temperature and the solvent was removed under vacuum. The crude was purified by flash column chromatography on $\mathrm{SiO}_{2}$, using $\mathrm{CS}_{2} /$ Toluene and then Toluene/THF (2:1). The black solids obtained was further purified by repeated 
(3x) precipitation and centrifugation in methanol to yield the corresponding hybrids as black solids. ${ }^{1} \mathrm{H}$ NMR (DMSO-d6, $\left.300 \mathrm{MHz}, 298 \mathrm{~K}\right) \delta 13.0$ (s, 1H), 8.25-7.59 (m), 5.02-4.56 (m), 2.90-2.60 (m).MS-ESI m/z 950.1 [M+H] .

Solar Cell Fabrication: Fluorine doped tin oxide (FTO) coated glass substrates (Pilkington TEC15, 15

substrates were sonicated for 15 minutes in a solution of milliQ water and soap (Hellmanex), rinsed with milliQ water, sonicated for 15 minutes in a solution of ethanol, rinsed with acetone and dried with compressed air. After that, a UV/ozone treatment was performed for 15 minutes. $\mathrm{TiO}_{2}$ compact layer was deposited onto the substrates by spray pyrolysis at $450^{\circ} \mathrm{C}$, using titanium diisopropoxidebis(acetylacetonate) in ethanol (1:39, v/v), with oxygen as carrier gas. Mesoporous $\mathrm{TiO}_{2}$ layer was deposited by spin coating at $6000 \mathrm{rpm}$ during $40 \mathrm{~s}$ using $\mathrm{TiO}_{2}$ paste diluted in ethanol (1:3.5, weight ratio). After drying at $80{ }^{\circ} \mathrm{C} 10 \mathrm{~min}$, it was heated at $470{ }^{\circ} \mathrm{C}$ for $30 \mathrm{~min}$ and cooled down to room temperature. The different fullerene derivatives were dissolved in chlorobenzene $(2 \mathrm{mg} / \mathrm{mL})$ and filtered with a $0.2 \mu \mathrm{m}$ PTFE filter. The substrates were heated $10 \mathrm{~min}$ at $120{ }^{\circ} \mathrm{C}$, immersed in these solutions for 24 hours, rinsed with chlorobenzene and then dried for $10 \mathrm{~min}$ at $120{ }^{\circ} \mathrm{C}$. The perovskite solution was prepared by mixing $2.64 \mathrm{M}$ of methylammonium iodide and $0.88 \mathrm{M}$ of lead chloride at a 3:1 mol ratio in DMF. The substrates were heated 10 min at $60^{\circ} \mathrm{C}$ and then the solution was spin-coated at $500 \mathrm{rpm}$ for $5 \mathrm{~s}$ and $2000 \mathrm{rpm}$ for $60 \mathrm{~s}$ in air conditions. After the deposition, the substrates were heated at $100{ }^{\circ} \mathrm{C}$ during 90 min in an oven under air stream. Spiro-OMeTAD was deposited by spin coating at $4000 \mathrm{rpm}$ for $30 \mathrm{~s}$. The spiro-OMeTAD solution was prepared by dissolving $72.3 \mathrm{mg}$ of spiro-OMeTAD, $28.8 \mu \mathrm{L}$ of TBP and $17.5 \mu \mathrm{L}$ of a stock solution of $520 \mathrm{mg} / \mathrm{mL}$ of LiTFSI in acetonitrile, in chlorobenzene. The deposition of $60 \mathrm{~nm}$ of gold was carried out 
by thermal evaporation at $10^{-6}$ mbar. Samples were prepared under air conditions and the humidity can vary in our laboratory between 30-60 \% and consequently samples prepared with different conditions cannot be straightforward compared among them. Our approach is to prepare reference samples for all the batches and compare each sample with its respective reference, see Table 1.

Characterization: The current-voltage curves were measured with a scan rate of 50 mV/s in Abet Technologies Sun 2000 Class A solar simulator with a Keithley 2612 Source Meter, AM1.5G and $100 \mathrm{~mW} \cdot \mathrm{cm}^{-2}$. The measurements were performed using a shadow mask whose area was $0.089 \mathrm{~cm}^{2}$.For the PL measurements, a commercial red laser diode $(650 \mathrm{~nm})$ was used as excitation source. The measurements were carried out using a spectrophotometer CCD based detector (charge-coupled device, AndoriDUS DV 420A-OE) coupled with a spectrograph as a diffraction grating (Newport 77400).

\section{Acknowledgements}

The work was supported by European Research Council (ERC-320441-Chirallcarbon), the CAM (FOTOCARBON project S2013/MIT-2841), MINECO of Spain (projects MAT2013-47192-C3-1-R, CTQ2014-52045-R and project MAT2015-70611-ERC) and by Generalitat Valenciana (Project PROMETEOII/2014/020). We acknowledge SCIC from University Jaume I for help with SEM characterization. B.C.H is grateful to the support of the National Council of Technological and Scientific Development (CNPq), Brazil, through the Science without Borders program.

\section{Supporting Information}

Supporting Information is available from the Wiley Online Library or from the author. 
Normalized absorbance spectra and cyclic voltammetry measurements of the fullerene

derivatives. Photoluminescence spectra and dark current of the complete devices.

\section{References}

(1) Kim, H.-S.; Lee, C.-R.; Im, J.-H.; Lee, K.-B.; Moehl, T.; Marchioro, A.; Moon, S.-J.; Humphry-Baker, R.; Yum, J.-H.; Moser, J. E. et al. Lead Iodide Perovskite Sensitized All-Solid-State Submicron Thin Film Mesoscopic Solar Cell with Efficiency Exceeding 9\%. Sci. Rep. 2012, 2, 591.

(2) Lee, M. M.; Teuscher, J.; Miyasaka, T.; Murakami, T. N.; Snaith, H. J. Efficient Hybrid Solar Cells Based on Meso-Superstructured Organometal Halide Perovskites. Science 2012, 338, 643-647.

(3) Yang, W. S.; Noh, J. H.; Jeon, N. J.; Kim, Y. C.; Ryu, S.; Seo, J.; Seok, S. I. High-Performance Photovoltaic Perovskite Layers Fabricated through Intramolecular Exchange. Science 2015, 348, 1234-1237.

(4) Jeon, N. J.; Noh, J. H.; Yang, W. S.; Kim, Y. C.; Ryu, S.; Seo, J.; Seok, S. I. Compositional Engineering of Perovskite Materials for High-Performance Solar Cells. Nature 2015, 517, 476-480.

(5) Jeon, N. J.; Noh, J. H.; Kim, Y. C.; Yang, W. S.; Ryu, S.; Seok, S. I. Solvent Engineering for High-Performance Inorganic-Organic Hybrid Perovskite Solar Cells. Nat. Mater. 2014, 13, 897-903.

(6) Saliba, M.; Matsui, T.; Seo, J.-Y.; Domanski, K.; Correa-Baena, J.-P.; Nazeeruddin, M. K.; Zakeeruddin, S. M.; Tress, W.; Abate, A.; Hagfeldt, A. et al. Cesium-Containing Triple Cation Perovskite Solar Cells: Improved Stability, Reproducibility and High Efficiency. Energy Environ. Sci. 2016, 9, 1989-1997.

(7) Molina-Ontoria, A.; Zimmermann, I.; Garcia-Benito, I.; Gratia, P.; Roldán-Carmona, C.; Aghazada, S.; Graetzel, M.; Nazeeruddin, M. K.; Martín, N. Benzotrithiophene-Based Hole-Transporting Materials for $18.2 \%$ Perovskite Solar Cells. Angew. Chem. Int. Ed. 2016, 55, 6270-6274.

(8) Green, M. A.; Emery, K.; Hishikawa, Y.; Warta, W.; Dunlop, E. D. Solar Cell Efficiency Tables (version 48). Prog. Photovolt: Res. Appl. 2016, 24, 905-913.

(9) Kazim, S.; Nazeeruddin, M. K.; Grätzel, M.; Ahmad, S. Perovskite as Light Harvester: A Game Changer in Photovoltaics. Angew. Chem. Int. Ed. 2014, 53, 2812-2824.

(10) Chen, B.; Yang, M.; Priya, S.; Zhu, K. Origin of J-V Hysteresis in Perovskite Solar Cells. J. Phys. Chem. Lett. 2016, 7, 905917.

(11) Levine, I.; Nayak, P. K.; Wang, J. T.-W.; Sakai, N.; van Reenen, S.; Brenner, T. M.; Mukhopadhyay, S.; Snaith, H. J.; Hodes, G.; Cahen, D. InterfaceDependent Ion Migration/Accumulation Controls Hysteresis in MAPbI3 Solar Cells. $J$. Phys. Chem. C 2016, 120, 16399-16411.

(12) Bryant, D.; Wheeler, S.; O’Regan, B. C.; Watson, T.; Barnes, P. R. F.; Worsley, D.; Durrant, J. Observable Hysteresis at Low Temperature in "Hysteresis Free” Organic-Inorganic Lead Halide Perovskite Solar Cells. J. Phys. Chem. Lett. 2015, 6, 3190-3194.

(13) Tress, W.; Marinova, N.; Moehl, T.; Zakeeruddin, S. M.; Nazeeruddin, M. K.; Gratzel, M. Understanding the Rate-Dependent J-V Hysteresis, Slow Time Component, and Aging in $\mathrm{CH}_{3} \mathrm{NH}_{3} \mathrm{PbI}_{3}$ Perovskite Solar Cells: The Role of a Compensated Electric Field. Energy Environ. Sci. 2015, 8, 995-1004. 
(14) Almora, O.; Aranda, C.; Zarazua, I.; Guerrero, A.; Garcia-Belmonte, G. Noncapacitive Hysteresis in Perovskite Solar Cells at Room Temperature. ACS Energy Lett. 2016, 1, 209-215.

(15) Bisquert, J.; Cahen, D.; Hodes, G.; Rühle, S.; Zaban, A. Physical Chemical Principles of Photovoltaic Conversion with Nanoparticulate, Mesoporous Dye-Sensitized Solar Cells. J. Phys. Chem. B 2004, 108, 8106-8118.

(16) Völker, S. F.; Collavini, S.; Delgado, J. L. Organic Charge Carriers for Perovskite Solar Cells. ChemSusChem 2015, 8, 3012-3028.

(17) Stranks, S. D.; Eperon, G. E.; Grancini, G.; Menelaou, C.; Alcocer, M. J. P.; Leijtens, T.; Herz, L. M.; Petrozza, A.; Snaith, H. Electron-Hole Diffusion Lengths Exceeding 1 Micrometer in an Organometal Trihalide Perovskite Absorber. J. Science 2013, 342, 341-344.

(18) Xing, G.; Mathews, N.; Sun, S.; Lim, S. S.; Lam, Y. M.; Grätzel, M.; Mhaisalkar, S.; Sum, T. C. Long-Range Balanced Electron- and Hole-Transport Lengths in Organic-Inorganic $\mathrm{CH}_{3} \mathrm{NH}_{3} \mathrm{PbI}_{3}$. Science 2013, 342, 344-347.

(19) Tao, C.; Neutzner, S.; Colella, L.; Marras, S.; Srimath Kandada, A. R.; Gandini, M.; Bastiani, M. D.; Pace, G.; Manna, L.; Caironi, M. et al. 17.6\% Stabilized Efficiency in Low-Temperature Processed Planar Perovskite Solar Cells. Energy Environ. Sci. 2015, 8, 2365-2370.

(20) Li, Y.; Zhao, Y.; Chen, Q.; Yang, Y.; Liu, Y.; Hong, Z.; Liu, Z.; Hsieh, Y.-T.; Meng, L.; Li, Y. Multifunctional Fullerene Derivative for Interface Engineering in Perovskite Solar Cells. J. Am. Chem. Soc. 2015, 137, 15540-15547.

(21) Wojciechowski, K.; Stranks, S. D.; Abate, A.; Sadoughi, G.; Sadhanala, A.; Kopidakis, N.; Rumbles, G.; Li, C.-Z.; Friend, R. H.; Jen, A. K. Y. et al. Heterojunction Modification for Highly Efficient Organic-Inorganic Perovskite Solar Cells. ACS Nano 2014, 8, 12701-12709.

(22) Sanchez, R. S.; Gonzalez-Pedro, V.; Lee, J.-W.; Park, N.-G.; Kang, Y. S.; Mora-Sero, I.; Bisquert, J. Slow Dynamic Processes in Lead Halide Perovskite Solar Cells. Characteristic Times and Hysteresis. J. Phys. Chem. Lett. 2014, 5, 2357-2363.

(23) Almora, O.; Zarazua, I.; Mas-Marza, E.; Mora-Sero, I.; Bisquert, J.; Garcia-Belmonte, G. Capacitive Dark Currents, Hysteresis, and Electrode Polarization in Lead Halide Perovskite Solar Cells. J. Phys. Chem. Lett. 2015, 6, 1645-1652.

(24) Carrillo, J.; Guerrero, A.; Rahimnejad, S.; Almora, O.; Zarazua, I.; MasMarza, E.; Bisquert, J.; Garcia-Belmonte, G. Ionic Reactivity at Contacts and Aging of Methylammonium Lead Triiodide Perovskite Solar Cells. Adv. Energy Mater. 2016, 6, DOI: $10.1002 /$ aenm.201502246.

(25) Stumpp, M.; Ruess, R.; Horn, J.; Tinz, J.; Richter, C.; Schlettwein, D. IV Hysteresis of Methylammonium Lead Halide Perovskite Films on Microstructured Electrode Arrays: Dependence on Preparation Route and Voltage Scale. Phys. Status Solidi A 2016, 213, 38-45.

(26) Tress, W.; Correa Baena, J. P.; Saliba, M.; Abate, A.; Graetzel, M. Inverted Current-Voltage Hysteresis in Mixed Perovskite Solar Cells: Polarization, Energy Barriers, and Defect Recombination. Adv. Energy Mater. 2016, DOI: 10.1002/aenm.201600396.

(27) Christians, J. A.; Manser, J. S.; Kamat, P. V. Best Practices in Perovskite Solar Cell Efficiency Measurements. Avoiding the Error of Making Bad Cells Look Good. J. Phys. Chem. Lett. 2015, 6, 852-857.

(28) Bi, C.; Wang, Q.; Shao, Y.; Yuan, Y.; Xiao, Z.; Huang, J. Non-wetting surface-driven high-aspect-ratio crystalline grain growth for efficient hybrid perovskite solar cells. Nat. Commun. 2015, 6, 7747. 
(29) Juarez-Perez, E. J.; Wußler, M.; Fabregat-Santiago, F.; Lakus-Wollny, K.; Mankel, E.; Mayer, T.; Jaegermann, W.; Mora-Sero, I. Role of the Selective Contacts in the Performance of Lead Halide Perovskite Solar Cells. J. Phys. Chem. Lett. 2014, 5, 680-685. 